\title{
Collocation Methods for Nonlinear Volterra Integro-Differential Equations with Infinite Delay*
}

\author{
By Hermann Brunner
}

\begin{abstract}
In this paper we study the numerical solution of nonlinear Volterra integrodifferential equations with infinite delay by spline collocation and related Runge-Kutta type methods. The kernel function in these equations is of the form $k(t, s, y(t), y(s))$, with a representative example given by Volterra's population equation, where we have $k(t, s, y(t), y(s))=a(t-s) \cdot G(y(t), y(s))$.
\end{abstract}

1. Introduction. This paper is concerned with the numerical solution of nonlinear Volterra integro-differential equations (VIDE's) with infinite delay,

$$
y^{\prime}(t)=f(t, y(t))+\int_{-\infty}^{t} k(t, s, y(t), y(s)) d s, \quad t \in I:=[0, T],
$$

where on $(-\infty, 0]$ the solution $y$ is to agree with a given initial function $\phi$ :

$$
y(t)=\phi(t), \quad-\infty<t \leq 0 .
$$

In many important applications the kernel function $k$ in (1.1a) is of the form

$$
k(t, s, y, z)=a(t-s) \cdot G(y, z),
$$

where the convolution part $a$ is a smooth (nonpositive) function. We mention two important examples:

(i) Volterra's population equation (cf. Volterra [19]-[21], Miller [11], [12, pp. 130140], and Cushing [5]). Here, we have

$$
k(t, s, y, z)=a(t-s) \cdot y \cdot z,
$$

where typically

$$
a(t)=-\left(\gamma_{0} b^{-1}+\gamma_{1} b^{-2} t\right) \cdot \exp (-t / b)
$$

$\left(b>0, \gamma_{0}+\gamma_{1}=1, \gamma_{1}>\gamma_{0} \geq 0\right)$. Moreover,

$$
f(t, y)=y \cdot\left(a_{0}-a_{1} y\right), \quad \text { with } a_{0}, a_{1}>0 .
$$

(ii) VIDE of polymer rheology (cf. Lodge et al. [9], and Markowich and Renardy [10]). In this case, the kernel function $k$ is given by

$$
k(t, s, y, z)=a(t-s) \cdot\left(y^{3} / z^{2}-z\right),
$$

Received April 15, 1988.

1980 Mathematics Subject Classification (1985 Revision). Primary 65R99, 45J05; Secondary $92 \mathrm{~A} 15$.

Key words and phrases. Volterra integro-differential equations, infinite delay, Volterra's population equation, spline collocation, implicit Runge-Kutta type methods.

${ }^{*}$ This research was supported by the Natural Sciences and Engineering Research Council of Canada (NSERC Operating Grant No. A9406). 
with

$$
a(t)=-\mu^{-1} \cdot \sum_{k=1}^{r} \gamma_{k} \cdot \exp \left(-\lambda_{k} t\right)
$$

$\left(\gamma_{k}, \lambda_{k}>0,0<\mu \ll 1\right)$.

The existence and uniqueness of solutions to (1.1a), (1.1b), or to the equivalent initial value problem

$$
\begin{aligned}
& y^{\prime}(t)=f(t, y(t))+(Q y)(t)+\int_{0}^{t} k(t, s, y(t), y(s)) d s, \quad t \in I \\
& y(0)=\phi(0)
\end{aligned}
$$

where

$$
(Q y)(t):=\int_{-\infty}^{0} k(t, s, y(t), \phi(s)) d s, \quad t \in I,
$$

has been studied extensively in recent years. We refer in particular to the comprehensive survey paper by Corduneanu and Lakshmikantham [4] and its extensive list of references; compare also, e.g., [11], [9], [8], [3], [22], and [18].

In view of later applications (see Section 4) we cite a specific result concerning Volterra's population equation, i.e., (1.1a), (1.1b) with $f$ and $k$ given by (1.3c) and (1.3a), respectively.

THEOREM 1.1 (Miller [11]). Suppose that $a_{0}>0, a_{1}>0$, and let $a \in C[0, \infty) \cap$ $L^{1}[0, \infty)$, with $a(t) \not \equiv 0$, satisfy

$$
a_{1}-\int_{0}^{\infty}|a(s)| d s>0 .
$$

Then for any positive, continuous, bounded function $\phi(t),-\infty<t \leq 0$, the problem (1.1a), (1.1b), with $f$ and $k$ given by (1.3c) and (1.3a), respectively, has a unique solution $y \in C^{1}[0, \infty)$. This solution satisfies $y(t)>0$ for all $t>0$, and we have

$$
\lim _{t \rightarrow \infty} y(t)=a_{0} /\left(a_{1}-\int_{0}^{\infty} a(s) d s\right) .
$$

In the following we will always assume that the problem (1.1a), (1.1b) possesses a unique solution $y$ on the interval $I$.

The systematic numerical treatment of VIDE's with infinite delay (or even the classical initial value problem for VIDE's whose kernel function $k$ depends both on $y(t)$ and $y(s)$, with $0 \leq s \leq t$ ) has received very little attention in the literature. Pouzet dedicated one part of his thesis [15] to the numerical solution of a higherorder version of the initial value problem $(1.5 \mathrm{a})$ (with $(Q y)(t) \equiv 0$ : no delay): the given VIDE is rewritten as a system of second-kind Volterra integral equations, and this system is then solved by adapting the classical 4-stage, explicit RungeKutta method for ordinary differential equations. (Computer programs based on this approach may be found in [16].) However, this early approach does not seem to have been followed up.

More recently, a certain class of VIDE's with infinite delay arising as model equations describing the stretching of polymeric liquids, i.e., (1.1a) with kernel function (1.4a), were solved by means of first-order implicit Euler type discretizations 
(a special case of Radau II type discretization; cf. Section 3, (3.5)). This specific way of treating the problem was dictated by the particular qualitative behavior of the exact solution (see [13], [10]).

The present paper is organized as follows. In Section 2 we introduce polynomial spline collocation methods for VIDE's with infinite delay and analyze their convergence properties. Section 3 deals with the numerical implementation of these methods: since the various integrals occurring in the underlying collocation equation cannot, in general, be found analytically, they will have to be approximated by appropriate quadrature processes, leading to implicit methods of Runge-Kutta type. As we have already mentioned, Section 4 contains the application of these methods to Volterra's population equation. A number of open problems are discussed in Section 5.

2. Collocation Approximations in Continuous Spline Spaces. Let $\Pi_{N}$ : $0=t_{0}<t_{1}<\cdots<t_{N}=T(N \geq 1)$ be a partition (mesh) of the given compact interval $I:=[0, T]$, and set $\sigma_{n}:=\left[t_{n}, t_{n+1}\right], h_{n}:=t_{n+1}-t_{n}(n=0, \ldots, N-1)$, and $h:=\max \left\{h_{n}: 0 \leq n \leq N-1\right\}$ (mesh diameter). Moreover, denote by $\pi_{m}$ the set of all real polynomials of degree not exceeding $m$. The exact solution $y$ of (1.5a), (1.5b) (referred to subsequently as (1.5)) will be approximated on $I$ by an element of the space

$$
S_{m}^{(0)}\left(\Pi_{N}\right):=\left\{u \in C(I):\left.u\right|_{\sigma_{n}}=: u_{n} \in \pi_{m}(0 \leq n \leq N-1)\right\}
$$

i.e., by a continuous polynomial spline function of degree $m$. Obviously,

$$
\operatorname{dim} S_{m}^{(0)}\left(\Pi_{N}\right)=N m+1
$$

This approximation $u$ will be determined by collocation: if

$$
X\left(\Pi_{N}\right):=\bigcup_{n=0}^{N-1} X_{n}
$$

with

$$
X_{n}:=\left\{t=t_{n, j}:=t_{n}+c_{j} h_{n}: 0 \leq c_{1}<\cdots<c_{m} \leq 1\right\},
$$

is a given set of collocation points in $I$ (based on the collocation parameters $\left\{c_{j}\right\}$ ), then $u$ is to satisfy $(1.5)$ on this set $X\left(\Pi_{N}\right)$ :

$$
u^{\prime}(t)=f(t, u(t))+(Q u)(t)+\int_{0}^{t} k(t, s, u(t), u(s)) d s, \quad t \in X\left(\Pi_{N}\right)
$$

with $u(0)=\phi(0)$ and

$$
(Q u)(t):=\int_{-\infty}^{0} k(t, s, u(t), \phi(s)) d s .
$$

Note that if $c_{1}=0$ and $c_{m}=1$ (i.e., $t_{n-1, m}=t_{n, 1}=t_{n}, n=1, \ldots, N$ ), if $f$ and $k$ are continuous on their respective domains, and if the collocation approximation $u$ exists, then it satisfies

$$
u \in S_{m}^{(0)}\left(\Pi_{N}\right) \cap C^{1}(I)=: S_{m}^{(1)}\left(\Pi_{N}\right) .
$$


Since $u_{n}^{\prime}$ (the restriction of $u^{\prime}$ to the subinterval $\sigma_{n}$ ) is in $\pi_{m-1}$, it can be expressed in the form

$$
u_{n}^{\prime}\left(t_{n}+v h_{n}\right)=\sum_{k=1}^{m} L_{k}(v) Y_{n, k}
$$

where $Y_{n, k}:=u_{n}^{\prime}\left(t_{n, k}\right)$ and $L_{k}(v):=\prod_{l=1, l \neq k}^{m}\left(v-c_{l}\right) /\left(c_{k}-c_{l}\right)$. Hence, setting

$$
a_{k}(v):=\int_{0}^{v} L_{k}(z) d z \quad(k=1, \ldots, m)
$$

we obtain

$$
u_{n}\left(t_{n}+v h_{n}\right)=y_{n}+h_{n} \cdot \sum_{k=1}^{m} a_{k}(v) Y_{n, k}, \quad v \in[0,1],
$$

with $y_{n}:=u_{n}\left(t_{n}\right)\left(=u_{n-1}\left(t_{n}\right)\right)$ and $y_{0}=y(0)=\phi(0)$. Using these representations in the collocation equation $(2.2 \mathrm{a})$, we are led to

$$
\begin{aligned}
Y_{n, j}= & f\left(t_{n, j}, U_{n, j}\right)+(Q u)\left(t_{n, j}\right) \\
& +h_{n} \cdot \int_{0}^{c_{j}} k\left(t_{n, j}, t_{n}+v h_{n}, U_{n, j}, y_{n}+h_{n} \cdot \sum_{k=1}^{m} a_{k}(v) Y_{n, k}\right) d v \\
& +\sum_{i=0}^{n-1} h_{i} \cdot \int_{0}^{1} k\left(t_{n, j}, t_{i}+v h_{i}, U_{n, j}, y_{i}+h_{i} \cdot \sum_{k=1}^{m} a_{k}(v) Y_{i, k}\right) d v \\
& (j=1, \ldots, m ; n=0, \ldots, N-1),
\end{aligned}
$$

with

$$
U_{n, j}:=u_{n}\left(t_{n, j}\right)=y_{n}+h_{n} \cdot \sum_{k=1}^{m} a_{j, k} Y_{n, k} \quad\left(a_{j, k}:=a_{k}\left(c_{j}\right)\right)
$$

and

$$
(Q u)\left(t_{n, j}\right)=\int_{-\infty}^{0} k\left(t_{n, j}, s, U_{n, j}, \phi(s)\right) d s .
$$

For each $n=0, \ldots, N-1$, (2.6a) represents a system of nonlinear equations in $\mathbb{R}^{m}$. Once the vectors $Y_{n}:=\left(Y_{n, 1}, \ldots, Y_{n, m}\right)^{T}(0 \leq n \leq N-1)$ are known, the collocation solution $u \in S_{m}^{(0)}\left(\Pi_{N}\right)$ is completely determined by (2.5).

It is readily verified that, under appropriate assumptions on $f, k$, and $\phi$ in (1.1) (assuring the existence of a unique solution $y \in C^{1}[0, T]$ ), the Contraction Mapping Theorem (cf. [14, Chapter 12]) guarantees that each of the systems (2.6a) has a unique solution $Y_{n} \in \mathbb{R}^{m}$ whenever the mesh diameter, $h:=\max _{(n)}\left\{h_{n}\right\}$, is sufficiently small.

In the following we shall focus on results dealing with the order of convergence of $u$ at the mesh points $Z_{N}:=\left\{t_{n}: 1 \leq n \leq N\right\}$. However, for the sake of comparison we also state a global convergence result; its proof, which we omit, relies on standard techniques (compare [1], [2, pp. 280-284]). In all subsequent theorems it is assumed that the partitions $\Pi_{N}(N \in \mathbb{N})$ are quasi-uniform, i.e., the quotients $h / \bar{h}$, with $\bar{h}:=\min \left\{h_{n}: 0 \leq n \leq N-1\right\}$, are uniformly bounded:

$$
h / \bar{h} \leq \gamma, \quad N \in \mathbb{N},
$$

for some constant $\gamma \geq 1$. If all partitions are uniform $\left(h_{n}=h=T N^{-1}\right.$ for all $\left.n\right)$ then, of course, $\gamma=1$. Note that (2.7) implies $N h \leq \gamma T<\infty$. 
THEOREM 2.1. Let $f$ and $k$ in (1.1) have continuous derivatives of order $m$ and be such that, for given $\phi \in C(-\infty, 0]$, the initial value problem (1.5a) has a unique solution $y \in C^{m+1}(I)$. If $u \in S_{m}^{(0)}\left(\Pi_{N}\right)$ denotes the collocation approximation defined by (2.6a), (2.5), then

$$
\left\|y^{(r)}-u^{(r)}\right\|:=\sup \left\{\left|y^{(r)}(t)-u^{(r)}(t)\right|: t \in I\right\}=O\left(N^{-m}\right) \quad(r=0,1)
$$

as $N \rightarrow \infty$, and this holds for any choice of the collocation points $X\left(\Pi_{N}\right)$ given by (2.1b).

While global convergence (on $I$ ) of order $p=m$ occurs for any choice of $m$ distinct collocation parameters $\left\{c_{j}\right\}$ in $[0,1]$, certain sets $\left\{c_{j}\right\}$ lead to a higher order of convergence, $p^{*}>m$, at the mesh points $Z_{N}$. This is an interesting property of polynomial spline collocation methods, since in many applications one is interested above all in obtaining highly accurate approximations to $y$ at certain specific mesh points (e.g., at $t_{N}=T$ ).

In the subsequent analysis, the integrals

$$
J_{k}:=\int_{0}^{1} s^{k} \cdot \prod_{j=1}^{m}\left(s-c_{j}\right) d s, \quad k \in \mathbb{N}_{0}
$$

will play a critical role.

THEOREM 2.2. Let $u \in S_{m}^{(0)}\left(\Pi_{N}\right)$ be the collocation approximation defined by (2.6a), (2.5), and assume that the collocation parameters $\left\{c_{j}\right\}$ have been chosen so that $J_{k}=0$ for $k=0, \ldots, d-1$, and $J_{d} \neq 0$ for some $d$ with $0 \leq d \leq m$. If $f$ and $k$ in (1.1) have continuous derivatives of order $m+d$ and are such that the initial value problem (1.5a) has, for given $\phi \in C(-\infty, 0]$, a unique solution $y \in C^{m+d+1}(I)$, then

$$
\max _{t_{n} \in Z_{N}}\left|y\left(t_{n}\right)-u\left(t_{n}\right)\right|=O\left(N^{-(m+d)}\right)
$$

while, in general, $\max _{t_{n} \in Z_{N}}\left|y^{\prime}\left(t_{n}\right)-u^{\prime}\left(t_{n}\right)\right|=O\left(N^{-m}\right)$ only.

If, however, $c_{m}=1$, then

$$
\max _{t_{n} \in Z_{N}}\left|y^{(r)}\left(t_{n}\right)-u^{(r)}\left(t_{n}\right)\right|=O\left(N^{-(m+d)}\right) \quad \text { for } r=0,1 .
$$

It is well known that the orthogonality conditions $J_{k}=0(k=0, \ldots, d-1)$ imply that the degree of precision of the $m$-point interpolatory quadrature formula on $[0,1]$ based on the abscissas $\left\{c_{j}\right\}$ has degree of precision $m+d-1$. Since this degree of precision cannot exceed the value $2 m-1$, we always have $d \leq m$. The following corollary deals with some important special cases, and its proof relies on the above observation.

COROLlary 2.1. Let $f, k$, and $\phi$ be as in Theorem 2.2, and let the collocation approximation $u \in S_{m}^{(0)}\left(\Pi_{N}\right)$ be defined by (2.6) and (2.5).

(a) If the collocation parameters $\left\{c_{j}\right\}$ are the $m$ Gauss(-Legendre) points for $(0,1)$, i.e., the zeros of the shifted Legendre polynomial $P_{m}(2 s-1)$, then $J_{k}=0$ for $k=0, \ldots, m-1$, with $J_{m} \neq 0$, and hence

$$
\max _{t_{n} \in Z_{N}}\left|y\left(t_{n}\right)-u\left(t_{n}\right)\right|=O\left(N^{-2 m}\right),
$$

while $\max _{t_{n} \in Z_{N}}\left|y^{\prime}\left(t_{n}\right)-u^{\prime}\left(t_{n}\right)\right|=O\left(N^{-m}\right)$ only. 
(b) The optimal order in (2.11), $m+d=2 m-1$, is attained if, and only if, the collocation parameters $\left\{c_{j}\right\}$ are the Radau II points, i.e., the zeros of $P_{m}(2 s-1)-$ $P_{m-1}(2 s-1)$.

(c) If the collocation approximation $u$ is to lie in the smoother spline space $S_{m}^{(1)}\left(\Pi_{N}\right)$, then the optimal order in $(2.11)$ is $m+d=2 m-2(m \geq 2)$. The corresponding collocation parameters $\left\{c_{j}\right\}$ are the Lobatto points, i.e., the zeros of $s(s-1) P_{m-1}^{\prime}(2 s-1)$.

If one selects equally spaced collocation parameters $\left\{c_{j}\right\}$, then the corresponding interpolatory quadrature formulas are the Newton-Cotes formulas. In this case, $d \in\{0,1\}$, with $d=1$ (and hence $m+d=m+1$ in (2.11)) if, and only if, $m$ is odd. The case $m=3$ (Simpson's Rule) coincides of course with the result contained in part (c) of Corollary 2.1.

Proof of Theorem 2.2. The collocation equation (2.2a) can be written in the form

$$
u^{\prime}(t)=f(t, u(t))+(Q u)(t)-\delta(t)+\int_{0}^{t} k(t, s, u(t), u(s)) d s, \quad t \in I,
$$

with $u(0)=\phi(0)$, where the residual function $\delta(t)$ vanishes at all collocation points:

$$
\delta(t)=0 \text { for all } t \in X\left(\Pi_{N}\right) .
$$

The proof of Theorem 2.2 will be based on the fact that the collocation error, $e(t):=y(t)-u(t)$, may be viewed as the solution of a nonlinearly perturbed VIDE.

LEMMA 2.1. Assume that $f(t, y)$ and $k(t, s, y, z)$ have continuous partial derivatives of order at least two with respect to $y$ and $z$. Then the collocation error corresponding to the collocation approximation $u \in S_{m}^{(0)}\left(\Pi_{N}\right)$ satisfies the initial value problem

$$
\begin{aligned}
& e^{\prime}(t)=P(t) e(t)+\delta(t)+\int_{0}^{t} H(t, s) e(s) d s+(\Phi e)(t), \quad t \in I \\
& e(0)=0
\end{aligned}
$$

where

$$
P(t):=f_{y}(t, y(t))+\int_{-\infty}^{t} k_{y}(t, s, y(t), y(s)) d s
$$

$($ with $y(s)=\phi(s)$ for $-\infty<s \leq 0)$,

$$
H(t, s):=k_{z}(t, s, y(t), y(s)),
$$

and

$$
\begin{aligned}
(\Phi e)(t):= & -\frac{1}{2}\left\{f_{y y}(t, \cdot)+\int_{-\infty}^{0} k_{y y}(t, s, \cdot, \phi(s)) d s\right. \\
& \left.+\int_{0}^{t} k_{y y}(t, s, \cdot, \cdot) d s\right\} e^{2}(t) \\
& -\int_{0}^{t} k_{y z}(t, s, \cdot, \cdot) e(s) d s \cdot e(t)-\frac{1}{2} \int_{0}^{t} k_{z z}(t, s, \cdot, \cdot) e^{2}(s) d s .
\end{aligned}
$$

Here, the unspecified arguments represent suitable functions of the form $y-\theta e$ $(0<\theta<1)$ arising in the remainder terms for Taylor's formula. 
Proof. It follows from (2.13) and (1.5a) that $e(t)$ satisfies the equation

$$
\begin{aligned}
e^{\prime}(t)= & (f(t, y(t))-f(t, u(t)))+((Q y)(t)-(Q u)(t))+\delta(t) \\
& +\int_{0}^{t}\{k(t, s, y(t), y(s))-k(t, s, u(t), u(s))\} d s, \quad t \in I .
\end{aligned}
$$

Setting $u=y-e$ and applying Taylor's formula,

$$
F(y-e)=F(y)-F^{\prime}(y) e+\frac{1}{2} F^{\prime \prime}(y-\theta e) e^{2}, \quad \text { with } 0<\theta<1,
$$

to the terms $f(t, y(t)-e(t)), k(t, s, y(t)-e(t), \phi(s)) \quad$ in $(Q u)(t))$, and $k(t, s, y(t)-e(t), y(s)-e(s))$, the assertion of Lemma 2.1 is readily verified.

Equation (2.15) represents a nonlinearly perturbed linear VIDE,

$$
e^{\prime}(t)=P(t) e(t)+\delta(t)+\int_{0}^{t} H(t, s) e(s) d s, \quad t \in I,
$$

where the perturbation term, $(\Phi e)(t)$, is given by (2.16). Nonlinear variation of parameter formulas for such VIDE's, as given in [17] (see also the list of references to earlier papers given therein), could be employed to obtain a representation for the solution $e(t)$ of (2.15). Here, however, we will use a somewhat different approach, as indicated in the following lemma.

LEMMA 2.2 (Grossman and Miller [7]). Let $R(t, s), 0 \leq s \leq t \leq T$, denote the solution of the resolvent equation associated with the linear part of the VIDE (2.15),

$$
\frac{\partial R(t, s)}{\partial s}=-R(t, s) P(s)-\int_{s}^{t} R(t, v) H(v, s) d v,
$$

satisfying $R(t, t)=1$ for all $t \in I$. Then $e(t)$ is a solution of (2.15) if, and only if, it satisfies the equation

$$
e(t)=R(t, 0) e(0)+\int_{0}^{t} R(t, s) \delta(s) d s+\int_{0}^{t} R(t, s)(\Phi e)(s) d s, \quad t \in I .
$$

Since the collocation error is subject to the initial condition $e(0)=0$, it solves the equation

$$
e(t)=\int_{0}^{t} R(t, s) \delta(s) d s+\int_{0}^{t} R(t, s)(\Phi e)(s) d s, \quad t \in I .
$$

Note, incidentally, that the resolvent $R(t, s)$ depends only on the given VIDE (1.1) (as indicated by the resolvent equation and the definition of the functions $P(t)$ and $H(t, s)$ in Lemma 2.1 , its smoothness properties are governed by those of the given functions $f, k$, and $\phi)$. The other terms on the right-hand side of $(2.17), \delta(s)$ and $(\Phi e)(s)$, reflect the global convergence properties of the collocation method (cf. Theorem 2.1): by $(2.8), \delta(t)$ is uniformly bounded for all sufficiently small mesh diameters $h>0$; moreover, by (2.13) it is piecewise smooth on $I$, provided $f, k$, and $\phi$ are smooth functions.

Now let $t=t_{n} \in Z_{N}$ in (2.17), and rewrite the first term on its right-hand side as

$$
\int_{0}^{t_{n}} R\left(t_{n}, s\right) \delta(s) d s=\sum_{i=0}^{n-1} h_{i} \int_{0}^{1} D_{n}\left(t_{i}+v h_{i}\right) d v
$$


where we have set $D_{n}\left(t_{i}+v h_{i}\right):=R\left(t_{n}, t_{i}+v h_{i}\right) \delta\left(t_{i}+v h_{i}\right)$. Note that these integrands, by (2.14), vanish if $v=c_{l}(l=1, \ldots, m)$. This observation suggests that we replace each of the integrals over $[0,1]$ by the $m$-point interpolatory quadrature formula having the $\left\{c_{l}\right\}$ as abscissas: if $E_{n, i}$ denotes the corresponding quadrature error, then

$$
\int_{0}^{1} D_{n}\left(t_{i}+v h_{i}\right) d v=\sum_{l=1}^{m} w_{l} D_{n}\left(t_{i}+c_{l} h_{i}\right)+E_{n, i}=E_{n, i} \quad(0 \leq i<n \leq N) .
$$

Thus, (2.17) at $t=t_{n}$ assumes the form

$$
e\left(t_{n}\right)=\sum_{i=0}^{n-1} h_{i} E_{n, i}+\int_{0}^{t_{n}} R\left(t_{n}, s\right)(\Phi e)(s) d s, \quad t_{n} \in Z_{N} .
$$

This in turn implies

$$
\left|e\left(t_{n}\right)\right| \leq h N \cdot \max _{(i, n)}\left|E_{n, i}\right|+B_{0} \cdot\|\Phi e\|
$$

where the constant $B_{0}$ is such that $\int_{0}^{t}|R(t, s)| d s \leq B_{0}$ for all $t \in I$, and where $(\Phi e)(t)$ is defined by $(2.16)$. Note that since the mesh sequence $\left\{\Pi_{N}\right\}$ is assumed to be quasi-uniform (cf. (2.7)), we have $N h \leq \gamma T<\infty$. Consider first $\|\Phi e\|$ : if the partial derivatives in (2.16) are bounded, and if the improper integral exists, then there is a constant $C_{0}$ so that

$$
\|\Phi e\|=\sup \{|(\Phi e)(t)|: t \in I\} \leq C_{0}\|e\|^{2} .
$$

By Theorem 2.1, $\|e\| \leq C_{1} h^{m}$ for all sufficiently small mesh diameters $h>0$ and all choices of the collocation parameters $\left\{c_{j}\right\}$. Hence,

$$
\|\Phi e\|=O\left(N^{-2 m}\right)
$$

since $h \leq \gamma T N^{-1}$.

Consider now the quadrature errors $E_{n, i}$ in (2.18). Since the corresponding quadrature formulas have degree of precision $m+d-1$ (recall (2.9) and the hypothesis in Theorem 2.2), it follows from Peano's Theorem (see, e.g., [6, pp. 285-292]) that

$$
\left|E_{n, i}\right| \leq \max _{0 \leq v \leq 1}\left|\frac{d^{m+d} D_{n}\left(t_{i}+v h_{i}\right)}{d v^{m+d}}\right| \cdot \int_{0}^{1}|K(s)| d s,
$$

where $K(s)$ denotes the Peano kernel of the quadrature formula. If the given functions in (1.1) are sufficiently smooth, then the integrands $D_{n}\left(t_{i}+v h_{i}\right)$ have the same degree of smoothness on $[0,1]$ and we have

$$
\left|\frac{d^{m+d} D_{n}\left(t_{i}+v h_{i}\right)}{d v^{m+d}}\right| \leq C_{2} h^{m+d}, \quad 0 \leq i<n \leq N
$$

for some constant $C_{2}$. Using the above estimates in (2.18) and recalling that $d \leq m$, we find

$$
\left|e\left(t_{n}\right)\right|=O\left(N^{-(m+d)}\right) \quad \text { for all } t_{n} \in Z_{N} .
$$

This establishes (2.10) in Theorem 2.2. 
In order to find an estimate for $e^{\prime}\left(t_{n}\right)$, differentiate (2.17) with respect to $t$. By Lemma 2.2 , the resolvent $R(t, s)$ satisfies $R(t, t)=1$ for all $t \in I$; moreover, $R(t, s)$ solves also the adjoint resolvent equation,

$$
\frac{\partial R(t, s)}{\partial t}=R(t, s) P(t)+\int_{s}^{t} H(t, v) R(v, s) d v, \quad 0 \leq s \leq t \leq T,
$$

with $R(s, s)=1$, thus ensuring the existence of the partial derivative $R_{t}(t, s)$. Thus,

$$
e^{\prime}(t)=\delta(t)+(\Phi e)(t)+\int_{0}^{t} R_{t}(t, s) \delta(s) d s+\int_{0}^{t} R_{t}(t, s)(\Phi e)(s) d s,
$$

$t \in I$. From this, an upper bound for the values $e^{\prime}\left(t_{m}\right)$ can be derived by an obvious modification of the above arguments, except that now the expression for $e^{\prime}\left(t_{n}\right)$ contains also the term $\delta\left(t_{n}\right)$ :

(i) If $t_{n}$ is a collocation point (i.e., if $c_{m}=1$ ), then $\delta\left(t_{n}\right)=0$ for all $t_{n} \in Z_{N}$, and hence $e^{\prime}\left(t_{n}\right)=O\left(N^{-(m+d)}\right)$ for all mesh points $Z_{N}$.

(ii) If $c_{m}<1$, then $t_{n} \notin X\left(\Pi_{N}\right)$, implying that, in general, $\delta\left(t_{n}\right) \neq 0$. It can be shown that in this case we only have

$$
\delta\left(t_{n}\right)=O\left(h^{m}\right), \quad t_{n} \in Z_{N}
$$

This proves the remaining assertions in Theorem 2.2.

3. Computational Forms of the Collocation Method. The collocation equations (2.6a) contain definite integrals which, in general, will have to be computed numerically by suitable quadrature processes. In order to avoid contamination of the order of convergence of the method, the degree of precision of the quadrature formulas has to be sufficiently large, namely at least $m+d-1$ (recall Theorem 2.2). Thus, a natural choice are the interpolatory quadrature formulas using the $m$ abscissas based on the collocation parameters $\left\{c_{j}\right\}$. For the integral over $\left[0, c_{j}\right]$ we have the approximation

$$
\int_{0}^{c_{j}} F_{n}\left(t_{n}+v h_{n}\right) d v \approx c_{j} \sum_{l=1}^{m} w_{l} F_{n}\left(t_{n}+c_{j} c_{l} h_{n}\right) \quad(j=1, \ldots, m)
$$

(note that the kernel function $k(t, s, y, z)$ is in general only given for $s \leq t$ ), while on $[0,1]$ we choose

$$
\int_{0}^{1} F_{n}\left(t_{i}+v h_{i}\right) d v \approx \sum_{l=1}^{m} w_{l} F_{n}\left(t_{i, l}\right) \quad\left(t_{i, l}=t_{i}+c_{l} h_{i}\right) .
$$

Here, $F_{n}\left(t_{i}+v h_{i}\right)(i \leq n)$ represents the respective integrand in (2.6a), and we have

$$
w_{l}:=\int_{0}^{1} \prod_{\substack{k=1 \\ k \neq l}}^{m}\left(s-c_{k}\right) /\left(c_{l}-c_{k}\right) d s .
$$

The remaining integrals are those occurring in the delay term (2.6c). In many applications these integrals can be expressed in "separable" form,

$$
(Q u)\left(t_{n, j}\right)=\sum_{i=1}^{r} \Psi_{i}\left(U_{n, j}\right) \cdot \int_{-\infty}^{0} K_{i}\left(t_{n, j}, s, \phi(s)\right) d s \quad(j=1, \ldots, m)
$$


where the integrals of $K_{i}(t, s, \phi(s))$ can be found analytically. For the particular kernels (1.3a) and (1.4a), the right-hand side of (3.1) is described, respectively, by

$$
\begin{array}{ll}
r=1 ; & \Psi_{1}(y)=y, K_{1}(t, s, z)=a(t-s) z ; \text { and } \\
r=2 ; & \Psi_{1}(y)=y^{3}, K_{1}(t, s, z)=a(t-s) z^{-2} \\
& \Psi_{2}(y)=-1, K_{2}(t, s, z)=a(t-s) z .
\end{array}
$$

If this is not possible, assume that $(Q u)\left(t_{n, j}\right)$ is approximated by

$$
(\hat{Q} u)\left(t_{n, j}\right):=\sum_{l=-M}^{0} b_{l} k\left(t_{n, j}, t_{l}, U_{n, j}, \phi\left(t_{l}\right)\right),
$$

where $M$ is either finite or $\infty$, and where the points $\left\{t_{l}\right\}$ are suitable quadrature abscissas, with corresponding weights $\left\{b_{l}\right\}$.

If the above quadrature processes are employed in $(2.6 \mathrm{a})$, then there results a fully discretized collocation method defining an element $\hat{u} \in S_{m}^{(0)}\left(\Pi_{N}\right)$ (which, in general, will be different from the approximation $u$ determined by the exact collocation equation (2.6a)):

$$
\begin{aligned}
\hat{Y}_{n, j}= & f\left(t_{n, j}, \hat{U}_{n, j}\right)+(\hat{Q} \hat{u})\left(t_{n, j}\right) \\
& +h_{n} c_{j} \sum_{l=1}^{m} w_{l} k\left(t_{n, j}, t_{n}+c_{j} c_{l} h_{n}, \hat{U}_{n, j}, \hat{y}_{n}+h_{n} \sum_{k=1}^{m} a_{k}\left(c_{j} c_{l}\right) \hat{Y}_{n, k}\right) \\
& +\sum_{i=0}^{n-1} h_{i} \sum_{l=1}^{m} w_{l} k\left(t_{n, j}, t_{i, l}, \hat{U}_{n, j}, \hat{U}_{i, l}\right) \quad(j=1, \ldots, m),
\end{aligned}
$$

with $\hat{y}_{n}:=\hat{u}_{n}\left(t_{n}\right)\left(=\hat{u}_{n-1}\left(t_{n}\right)\right), \hat{y}_{0}=\phi(0)$, and

$$
\hat{U}_{n, j}:=\hat{u}_{n}\left(t_{n, j}\right)=\hat{y}_{n}+h_{n} \cdot \sum_{k=1}^{m} a_{j, k} \hat{Y}_{n, k},
$$

with $a_{j, k}:=a_{k}\left(c_{j}\right)$ given by (2.4). Here, $(\hat{Q} \hat{u})\left(t_{n, j}\right)$ is given either by (3.2) or by the exact expression (3.1). The above method may be viewed as an $m$-stage implicit Runge-Kutta type method for the VIDE (1.1a).

Illustration. Take $\hat{u} \in S_{1}^{(0)}\left(\Pi_{N}\right)(m=1), 0 \leq c_{1} \leq 1$, and a uniform mesh $\Pi_{N}$ (i.e., $h_{n}=h=T N^{-1}$ for all $n$ ).

In this case we have (see (2.4))

$$
a_{1}(v)=v, \quad a_{1,1}=a_{1}\left(c_{1}\right)=c_{1}, \quad w_{1}=a_{1}(1)=1,
$$

and hence

$$
\hat{U}_{n, 1}=\hat{y}_{n}+h c_{1} \hat{Y}_{n, 1} \quad\left(\text { with } \hat{Y}_{n, 1}:=\hat{u}_{n}^{\prime}\left(t_{n, 1}\right), t_{n, 1}=t_{n}+c_{1} h\right)
$$

and (see (2.5))

$$
\hat{y}_{n+1}=\hat{y}_{n}+h \hat{Y}_{n, 1} \text {. }
$$

The value $\hat{Y}_{n, 1}$ is found by solving the single nonlinear equation ((3.3a) with $\left.m=1\right)$

$$
\begin{aligned}
\hat{Y}_{n, 1}= & f\left(t_{n, 1}, \hat{U}_{n, 1}\right)+(\hat{Q} \hat{u})\left(t_{n, 1}\right) \\
& +h c_{1} \cdot k\left(t_{n, 1}, t_{n}+c_{1}^{2} h, \hat{U}_{n, 1}, \hat{y}_{n}+h c_{1}^{2} \hat{Y}_{n, 1}\right) \\
& +h \sum_{i=0}^{n-1} k\left(t_{n, 1}, t_{i, 1}, \hat{U}_{n, 1}, \hat{U}_{i, 1}\right) .
\end{aligned}
$$


(i) $c_{1}=1$ (Radau II point). Suppose that (3.2) is given by

$$
(\hat{Q} \hat{u})\left(t_{n, 1}\right):=h \cdot \sum_{l=-\infty}^{0} k\left(t_{n, 1}, l h, \hat{U}_{n, 1}, \phi(l h)\right)
$$

(the composite (right) rectangular rule for equal subintervals of length $h=T N^{-1}$ ).

For this choice of $c_{1}$ we have $t_{n, 1}=t_{n+1}$ and

$$
\hat{U}_{n, 1}=\hat{y}_{n}+h \hat{Y}_{n, 1}=\hat{y}_{n+1} \text {. }
$$

Thus, replacing $\hat{Y}_{n, 1}$ by $h^{-1}\left(\hat{y}_{n+1}-\hat{y}_{n}\right),(3.4 \mathrm{~b})$ becomes

$$
\begin{aligned}
h^{-1}\left(\hat{y}_{n+1}-\hat{y}_{n}\right)= & f\left(t_{n+1}, \hat{y}_{n+1}\right)+(\hat{Q} \hat{u})\left(t_{n+1}\right) \\
& +h \cdot \sum_{i=0}^{n} k\left(t_{n+1}, t_{i+1}, \hat{y}_{n+1}, \hat{y}_{i+1}\right) \\
& (n=0, \ldots, N-1),
\end{aligned}
$$

with

$$
(\hat{Q} \hat{u})\left(t_{n+1}\right)=h \cdot \sum_{l=-\infty}^{0} k\left(t_{n+1}, l h, \hat{y}_{n+1}, \phi(l h)\right) .
$$

The equations (3.5a), (3.5b) represent the implicit Euler discretization for the initial value problem (1.5). This discretization was first introduced by Nevanlinna [13] to solve a VIDE arising in rheology (cf. (1.4a)); compare also [10]. Its (global and local) order of convergence is $p=1$.

(ii) $c_{1}=1 / 2$ (Gauss point). In this case we have $t_{n, 1}=t_{n}+\frac{h}{2}, \hat{U}_{n, 1}=\hat{y}_{n}+\frac{h}{2} \hat{Y}_{n, 1}$, and $(3.4 \mathrm{~b})$ becomes

$$
\begin{aligned}
\hat{Y}_{n, 1}= & f\left(t_{n}+\frac{h}{2}, \hat{U}_{n, 1}\right)+(\hat{Q} \hat{u})\left(t_{n}+\frac{h}{2}\right) \\
& +\frac{h}{2} k\left(t_{n}+\frac{h}{2}, t_{n}+\frac{h}{4}, \hat{U}_{n, 1}, \hat{y}_{n}+\frac{h}{4} \hat{Y}_{n, 1}\right) \\
& +h \cdot \sum_{i=0}^{n-1} k\left(t_{n}+\frac{h}{2}, t_{i}+\frac{h}{2}, \hat{U}_{n, 1}, \hat{U}_{i, 1}\right),
\end{aligned}
$$

with

$$
\hat{y}_{n+1}=\hat{y}_{n}+h \hat{Y}_{n, 1}
$$

Let $(\hat{Q} \hat{u})\left(t_{n}+\frac{h}{2}\right)$ be given either by

$$
(\hat{Q} \hat{u})\left(t_{n}+\frac{h}{2}\right)=h \cdot \sum_{l=-\infty}^{-1} k\left(t_{n}+\frac{h}{2},\left(l+\frac{1}{2}\right) h, \hat{U}_{n, 1}, \phi\left(\left(l+\frac{1}{2}\right) h\right)\right)
$$

(the composite midpoint rule), or by

$$
\begin{aligned}
& (\hat{Q} \hat{u})\left(t_{n}+\frac{h}{2}\right) \\
& \quad=h\left\{\sum_{l=-\infty}^{-1} k\left(t_{n}+\frac{h}{2}, l h, \hat{U}_{n, 1}, \phi(l h)\right)+\frac{1}{2} \cdot k\left(t_{n}+\frac{h}{2}, 0, \hat{U}_{n, 1}, \phi(0)\right)\right\}
\end{aligned}
$$

(the composite trapezoidal rule). The equations (3.6a), (3.6c) describe the midpoint method for the initial value problem (1.5). Under appropriate conditions on $k$ and $\phi$ 
(see also below) the approximations generated by (3.6a), (3.6c), or by $(3.6 \mathrm{a}),(3.7)$, converge quadratically at the mesh points:

$$
\max _{t_{n} \in Z_{N}}\left|y\left(t_{n}\right)-\hat{y}_{n}\right|=O\left(h^{2}\right) \text {. }
$$

We now return to the general method $(3.3 \mathrm{a})$ where the delay term is given by (3.2) or by (3.1). Setting $t=t_{n}+v h_{n}(v \in[0,1])$ we write (3.3a) in the analogous form of (2.13),

$$
\begin{aligned}
\hat{u}^{\prime}(t)= & f(t, \hat{u}(t))+(\hat{Q} \hat{u})(t)-\hat{\delta}(t) \\
& +h_{n} v \sum_{l=1}^{m} w_{l} k\left(t, t_{n}+v c_{l} h_{n}, \hat{u}(t), \hat{u}\left(t_{n}+v c_{l} h_{n}\right)\right) \\
& +\sum_{i=0}^{n-1} h_{i} \sum_{l=1}^{m} w_{l} k\left(t, t_{i, l}, \hat{u}(t), \hat{u}\left(t_{i, l}\right)\right), \quad t \in I
\end{aligned}
$$

with

$$
(\hat{Q} \hat{u})(t):=\sum_{l=-M}^{0} b_{l} k\left(t, t_{l}, u(t), \phi\left(t_{l}\right)\right),
$$

and with a residual function $\hat{\delta}(t)$ vanishing at the collocation points $X\left(\Pi_{N}\right)$. If we replace each of the quadrature expressions by the corresponding definite integral minus the induced quadrature error, $E_{i}^{n}(t)(i \leq n)$, and if we set

$$
(\hat{Q} \hat{u})(t)=(Q \hat{u})(t)-E_{-}(t)
$$

(with $(Q \hat{u})(t)$ as in $(2.2 \mathrm{~b})$, and with $E_{-}(t)$ denoting the quadrature error of the quadrature formula $(3.2))$, then we find

$$
\begin{aligned}
\hat{u}^{\prime}(t)= & f(t, \hat{u}(t))+(Q \hat{u})(t)-E_{-}(t)-\hat{\delta}(t) \\
& +\int_{0}^{t} k(t, s, \hat{u}(t), \hat{u}(s)) d s-E_{+}^{n}(t), \quad t=t_{n}+v h_{n} \in \sigma_{n},
\end{aligned}
$$

with $E_{+}^{n}(t):=\sum_{i=0}^{n} E_{i}^{n}(t)$. Hence, subtracting (3.9) from (1.5a), setting $\hat{e}(t):=$ $y(t)-\hat{u}(t)$, and applying Lemma 2.1 , we find the error equation

$$
\begin{aligned}
& \hat{e}^{\prime}(t)=P(t) \hat{e}(t)+\hat{\delta}(t)+E_{-}(t)+E_{+}^{n}(t) \\
& \quad+\int_{0}^{t} H(t, s) \hat{e}(s) d s+(\Phi \hat{e})(t), \\
& \quad t=t_{n}+v h_{n} \in \sigma_{n}, 0 \leq n \leq N-1,
\end{aligned}
$$

with $\hat{e}(0)=0$.

The above nonlinearly perturbed VIDE (3.10) differs from (2.15) in that it contains the additional terms $E_{-}(t)$ and $E_{+}^{n}(t)$, i.e., perturbations due to the quadrature approximations used in the discretization process for (2.6a) and (2.6c). Thus, the application of Lemma 2.2 to (3.10) yields

$$
\begin{aligned}
\hat{e}(t)= & \int_{0}^{t} R(t, s) \hat{\delta}(s) d s+\int_{0}^{t} R(t, s)(\Phi \hat{e})(s) d s \\
& +\int_{0}^{t} R(t, s)\left(E_{-}(s)+E_{+}(s)\right) d s, \quad t \in I,
\end{aligned}
$$

where $E_{+}(t)=E_{+}^{n}(t)$ for $t \in \sigma_{n}$. 
THEOREM 3.1. Let the assumptions of Theorem 2.2 hold, and assume that the delay term $(Q \hat{u})(t)(c f .(2.2 \mathrm{~b}))$ can be evaluated analytically (i.e., in (3.8) we have $E_{-}(t)=0$ for all $\left.t \in I\right)$. Then the estimates $(2.10),(2.11)$ of Theorem 2.2 and the assertions of Corollary 2.1 hold also for the collocation approximation $\hat{u} \in S_{m}^{(0)}\left(\Pi_{N}\right)$ defined by the discretized collocation method (3.3a).

Proof. Since $E_{-}(t)=0$ for all $t \in I$, it can be shown that any choice of the collocation parameters $\left\{c_{j}\right\}$ leads to the global convergence result $\|\hat{e}\|_{\infty}=O\left(N^{-m}\right)$ (cf. Theorem 2.1). This is a consequence of the fact that any $m$-point interpolatory quadrature rule has degree of precision greater than, or equal to, $m-1$. Moreover, if the orthogonality conditions $(2.9)$ are satisfied for $k=0, \ldots, d-1$, then the quadrature formulas in (3.3a) all have the degree of precision $m+d-1$, i.e., the resulting quadrature errors $E_{i}^{n}(t)(0 \leq i \leq n \leq N-1)$ are $O\left(N^{-(m+d)}\right)$ for all sufficiently smooth integrands. The arguments used in the proof of Theorem 2.2 can then be applied to the expression (3.11) for the error $e(t)$, setting $t=t_{n} \in Z_{N}$.

If the delay term $(Q \hat{u})(t)$ cannot be evaluated analytically, then it follows from (3.11) and the arguments introduced in the proof of Theorem 2.2 that the order of the error $\hat{e}(t)$ at the mesh points $Z_{N}$ is given by

$$
\max _{t_{n} \in Z_{N}}\left|y\left(t_{n}\right)-\hat{u}\left(t_{n}\right)\right|=O\left(N^{-(m+d)}\right)+O\left(\left\|E_{-}\right\|_{\infty}\right) .
$$

Here, the size of the bound for $\left\|E_{-}\right\|_{\infty}$ depends on the behavior of the integrand on $(-\infty, 0]$. If this integrand, $k(t, s, u(t), \phi(s))$, is zero for $-\infty<s \leq-T_{0}$ and $t \in I$, for some $T_{0}>0$, then we can attain $\left\|E_{-}\right\|_{\infty}=O\left(N^{-(m+d)}\right)$ by using instead of (3.2) $m$-point interpolatory quadrature formulas (over subintervals of length $h$ ) whose abscissas are based on the collocation parameters $\left\{c_{j}\right\}$.

In the general case, it is possible to obtain $\left\|E_{-}\right\|_{\infty}=O\left(h^{q}\right)$, with $q \geq m+d$, by means of the composite trapezoidal rule (see also (3.7)),

$$
(\hat{Q} \hat{u})(t):=h\left\{\sum_{l=-\infty}^{-1} k(t, l h, \hat{u}(t), \phi(l h))+\frac{1}{2} k(t, 0, \hat{u}(t), \phi(0))\right\},
$$

provided the integrand, $H(s):=k(\cdot, s, \hat{u}(\cdot), \phi(s))$, possesses odd-order derivatives up to order $2 r-1$ which vanish at 0 and $-\infty: H^{(2 j-1)}(0)=H^{(2 j-1)}(-\infty)=$ $0, j=1, \ldots, r$. It is known (see, e.g., [6, pp. 208-210]) that we then have $\left\|E_{-}\right\|_{\infty} \leq C h^{2 r+1}$ for some finite constant $C$. Hence, if $r$ is such that $2 r+1$ at least matches $m+d-1$, the degree of precision of the $m$-point quadrature formulas based on the $\left\{c_{j}\right\}$, then $\hat{e}\left(t_{n}\right)=O\left(N^{-(m+d)}\right)$ on the mesh $\Pi_{N}$. (Similar results hold for the composite midpoint rule.) While in practical applications the condition $H^{(2 j-1)}(-\infty)=0, j=1, \ldots, r$, is usually satisfied, this is generally not true at $t=0$. In this case one will resort to the composite trapezoidal rule with end corrections at $t=0$, given by appropriate terms in the Euler-Maclaurin summation formula. Compare also [6, pp. 210-211] for other modifications of the trapezoidal rule.

4. Application: Volterra's Population Equation. In the case of Volterra's population equation,

$$
y^{\prime}(t)=F(y(t))+\int_{-\infty}^{t} a(t-s) y(t) y(s) d s, \quad t \in I,
$$


where

$$
y(t)=\phi(t), \quad-\infty<t \leq 0
$$

and

$$
F(y)=\left(a_{0}-a_{1} y\right) y \quad\left(a_{0}>0, a_{1}>0\right),
$$

the discretized collocation method (3.3a) (defining an approximation $\hat{u} \in S_{m}^{(0)}\left(\Pi_{N}\right)$ ) assumes the form

$$
\begin{array}{r}
\hat{Y}_{n, j}= \\
\left.+\hat{U}_{n, j}\right)+(Q \hat{u})\left(t_{n, j}\right) \\
+\hat{U}_{n, j}\left\{h_{n} c_{j} \cdot \sum_{l=1}^{m} w_{l} a\left(h_{n} c_{j}\left(1-c_{l}\right)\right) \cdot\left(\hat{y}_{n}+h_{n} \sum_{k=1}^{m} a_{k}\left(c_{j} c_{l}\right) \hat{Y}_{n, k}\right)\right. \\
\left.+\sum_{i=0}^{n-1} h_{i} \sum_{l=1}^{m} w_{l} a\left(t_{n, j}-t_{i, l}\right) \hat{U}_{i, l}\right\} \\
\quad(j=1, \ldots, m),
\end{array}
$$

with

$$
(Q \hat{u})\left(t_{n, j}\right)=\hat{U}_{n, j} \cdot \int_{-\infty}^{0} a\left(t_{n, j}-s\right) \phi(s) d s
$$

and

$$
\hat{U}_{n, j}=\hat{y}_{n}+h_{n} \sum_{k=1}^{m} a_{j, k} \hat{Y}_{n, k}
$$

Here,

$$
a_{j, k}=a_{k}\left(c_{j}\right)=\int_{0}^{c_{j}} \prod_{\substack{l=1 \\ l \neq k}}^{m}\left(v-c_{l}\right) /\left(c_{k}-c_{l}\right) d v
$$

(cf. (2.4)). Once the solution of the nonlinear algebraic system (4.1a) is known, the collocation approximation $\hat{u}$ on the subinterval $\sigma_{n}$ is given by

$$
\hat{u}\left(t_{n}+v h_{n}\right)=\hat{y}_{n}+h_{n} \cdot \sum_{k=1}^{m} a_{k}(v) \hat{Y}_{n, k} ;
$$

in particular, we obtain

$$
\hat{y}_{n+1}=\hat{u}\left(t_{n}+h_{n}\right)=\hat{y}_{n}+h_{n} \cdot \sum_{k=1}^{m} w_{k} \hat{Y}_{n, k} \quad\left(\text { with } w_{k}=a_{k}(1)\right),
$$

where $\hat{y}_{0}=\phi(0)$.

As mentioned in Section 1, many models of population growth employ delay kernels reflecting the presence of some instantaneous effect on growth rate response, with delayed maximum effect, i.e.,

$$
a(t)=-\left(\gamma_{0} b^{-1}+\gamma_{1} b^{-2} t\right) \cdot \exp (-t / b),
$$

with $\gamma_{0}+\gamma_{1}=1, \gamma_{1}>\gamma_{0} \geq 0, b>0$. This function $a(t)$ attains its maximum at $t=b\left(\gamma_{1}-\gamma_{0}\right) / \gamma_{1}$, and we have $\int_{0}^{\infty}|a(t)| d t=1$. Hence, the integrals in (4.1b) can usually be calculated analytically, and the order of convergence of the approximations $\hat{y}_{n}$ defined by the discretized collocation method (4.1a)-(4.1d) is therefore 
described by Theorem 3.1 and Corollary 2.1 (with $\hat{u}$ replacing $u$ ). In the following numerical illustration the values of the above parameters were chosen as

$$
\gamma_{0}=0.05, \quad \gamma_{1}=0.95, \quad b=1.0 ; \quad a_{0}=14.0, \quad a_{1}=1.1 ;
$$

the initial function is $\phi(t)=\exp \left(\gamma_{2} t\right)$, with $\gamma_{2}=0.5$. The corresponding solution $y(t)$ rises from 1.0 at $t=0$ to about 11.7 near $t=0.5$ and then tends to the asymptotic value $y(\infty)=20 / 3$ (cf. Theorem 1.1). The approximating spline space is $S_{2}^{(0)}\left(\Pi_{N}\right)$ (i.e., $m=2$ ), and the collocation parameters are the Gauss points $\left(c_{1}=(3-\sqrt{3}) / 6, c_{2}=(3+\sqrt{3}) / 6\right)$ and the Radau II points $\left(c_{1}=1 / 3, c_{2}=1\right)$. The integral in (4.1b) was calculated analytically. The numerical results contained in Table 4.1 reveal that collocation at the Gauss points leads to a somewhat faster convergence rate than collocation at the Radau II points. However, as indicated in Corollary 2.1, the latter furnishes better convergence of the values approximating $y^{\prime}(t)$ with $t=t_{n} \in Z_{N}$. The computations were carried out in double precision on a VAX 8800 .

TABLE 4.1

\begin{tabular}{|c|c|c|c|c|c|}
\hline $\begin{array}{c}t_{n} \\
(T=4.0)\end{array}$ & $\begin{array}{c}N \\
(h=T / N)\end{array}$ & $\begin{array}{l}\text { Gaus } \\
\hat{u}\left(t_{n}\right)\end{array}$ & $\begin{array}{l}\text { points: } \\
\hat{u}^{\prime}\left(t_{n}\right)\end{array}$ & $\begin{array}{c}\text { Radau } \\
\hat{u}\left(t_{n}\right)\end{array}$ & $\begin{array}{l}\text { I points: } \\
\hat{u}^{\prime}\left(t_{n}\right)\end{array}$ \\
\hline \multirow[t]{3}{*}{0.3} & 40 & 10.1438 & 22.1377 & 10.1324 & 21.7513 \\
\hline & 80 & 10.1453 & 21.5796 & 10.1434 & 21.6455 \\
\hline & 160 & 10.1454 & 21.5986 & 10.1451 & 21.6285 \\
\hline \multirow[t]{3}{*}{0.5} & 40 & 11.6710 & -0.58348 & 11.6807 & -0.11591 \\
\hline & 80 & 11.6724 & -0.12895 & 11.6736 & -0.03755 \\
\hline & 160 & 11.6725 & -0.04816 & 11.6726 & -0.02752 \\
\hline \multirow[t]{3}{*}{1.0} & 40 & 10.3564 & -3.34695 & 10.3577 & -3.34136 \\
\hline & 80 & 10.3561 & -3.34099 & 10.3563 & -3.33961 \\
\hline & 160 & 10.3561 & -3.33972 & 10.3561 & -3.33937 \\
\hline \multirow[t]{3}{*}{2.0} & 40 & 7.56846 & -1.90745 & 7.56904 & -1.90831 \\
\hline & 80 & 7.56826 & -1.90745 & 7.56838 & -1.90764 \\
\hline & 160 & 7.56825 & -1.90749 & 7.56827 & -1.90753 \\
\hline \multirow[t]{3}{*}{4.0} & 40 & 6.45791 & 0.13490 & 6.45789 & 0.13445 \\
\hline & 80 & 6.45793 & 0.13453 & 6.45792 & 0.13441 \\
\hline & 160 & 6.45793 & 0.13441 & 6.45793 & 0.13441 \\
\hline
\end{tabular}

5. Concluding Remarks. If the VIDE (1.1a) is of the form

$$
\mu \cdot y^{\prime}(t)=f(t, y(t))+\int_{-\infty}^{t} k(t, s, y(t), y(s)) d s, \quad t \in I,
$$


with $0<\mu \ll 1$, then it may be regarded as a singular perturbation of the equation

$$
0=f(t, y(t))+\int_{-\infty}^{t} k(t, s, y(t), y(s)) d s
$$

where $y(t)=\phi(t)$ on $(-\infty, 0]$. Particular cases of (5.1) were analyzed in [9], [8] (with $f(t, y) \equiv 0$ ), and in [10] (with $f(t, y)=g(t) y^{\alpha}, 0<\alpha<3$ ); the kernel function $k(t, s, y, z)$ was the one given in (1.4a). In [13], [10] the numerical scheme (3.5a), (3.5b) (implicit Euler discretization) was used to generate numerical approximations to the solution of this VIDE, and it is conjectured in the second of these papers that higher-order Radau II discretizations will also successfully simulate the exponential decay of the solution of (5.1) (uniformly as $\mu \rightarrow 0$ ). Although the stability analysis of the collocation method (2.6) and its discretized counterpart (3.3) is an open problem, the result (2.11) and part (b) of Corollary 2.1 provide a first indication on why (discretized) collocation based on the Radau II points will be superior to collocation using the Gauss points when solving problems like (5.1): it yields "balanced" (same order) approximations to $y(t)$ and to its slope at the mesh points.

In this paper the given VIDE is considered on some compact interval $I=[0, T]$. In view of asymptotic results like the one mentioned in Theorem 1.1, it would be of interest to analyze the asymptotic properties of the spline collocation approximation $u$ on $[0, \infty)$ for a fixed stepsize $h>0$ and to derive, e.g., bounds for $\left|y\left(t_{n}\right)-u\left(t_{n}\right)\right|$ as $n \rightarrow \infty$, with $t_{n}=n h$ and $h>0$ fixed. As in [13] and [10], where this was investigated for implicit Euler type methods, such an analysis will have to show that, at least for certain judicious choices of the collocation parameters $\left\{c_{j}\right\}$, the collocation approximation $u \in S_{m}^{(0)}\left(\Pi_{N}\right)$ preserves the qualitative properties of the exact solution $y$ of the given VIDE. We shall pursue this challenging problem elsewhere.

\author{
Department of Mathematics and Statistics \\ Memorial University of Newfoundland \\ St. John's, Newfoundland A1C 5S7 \\ Canada \\ E-mail: hbrunner@mun.bitnet
}

1. H. BRUNNER, "Implicit Runge-Kutta methods of optimal order for Volterra integro-differential equations," Math. Comp., v. 42, 1984, pp. 95-109.

2. H. BRUNNER \& P. J. VAN DER HOUWEN, The Numerical Solution of Volterra Equations, CWI Monographs, Vol. 3, North-Holland, Amsterdam, 1986.

3. T. A. Burton, Stability and Periodic Solutions of Ordinary and Functional Differential Equations, Academic Press, New York, 1985.

4. C. CORdunEanU \& V. LAKShmikantham, "Equations with unbounded delay: a survey," Nonlinear Anal., v. 4, 1980, pp. 831-877.

5. J. M. Cushing, Integrodifferential Equations and Delay Models in Population Dynamics, Lecture Notes in Biomath., vol. 20, Springer-Verlag, Berlin and New York, 1977.

6. P. J. DAVIS \& P. RABinowitz, Methods of Numerical Integration, 2nd ed., Academic Press, London, 1984.

7. S. I. GRossman \& R. K. MilleR, "Perturbation theory for integrodifferential systems," J. Differential Equations, v. 8, 1970, pp. 457-474.

8. G. S. JORDAN, "A nonlinear singularly perturbed Volterra integrodifferential equation of nonconvolution type," Proc. Roy. Soc. Edinburgh Sect. A, v. 80, 1978, pp. 235-247. 
9. A. S. Lodge, J. B. MCLeod \& J. A. NOHEL, "A nonlinear singularly perturbed Volterra integrodifferential equation occurring in polymer rheology," Proc. Roy. Soc. Edinburgh Sect. A, v. 80,1978 , pp. $99-137$.

10. P. MARKOWICH \& M. RENARDY, "A nonlinear Volterra integrodifferential equation describing the stretching of polymer liquids," SIAM J. Math. Anal., v. 14, 1983, pp. 66-97.

11. R. K. Miller, "On Volterra's population equation," SIAM J. Appl. Math., v. 14, 1966, pp. 446-452.

12. R. K. Miller, Nonlinear Volterra Integral Equations, Benjamin, Menlo Park, CA, 1971.

13. O. Nevanlinna, Numerical Solution of a Singularly Perturbed Nonlinear Volterra Equation, MRC Tech. Sum. Report No. 1881, University of Wisconsin, Madison, 1978.

14. J. M. ORtegA \& W. C. Rheinboldt, Iterative Solution of Nonlinear Equations in Several Variables, Academic Press, New York, 1970.

15. P. Pouzet, Etude, en Vue de leur Approximation Numérique, des Solutions d'Équations Intégrales et Intégrodifférentielles de Type Volterra pour des Problèmes de Conditions Initiales, Ph.D. Thesis, University of Strasbourg, 1962.

16. P. Pouzet, "Systèmes différentiels, équations intégrales et intégrodifférentielles," in Procédures ALGOL en Analyse Numérique. I, Centre National de la Recherche Scientifique, Paris, 1967, pp. 203-208.

17. Shouchuan Hu, V. Lakshmikantham \& M. Rama Mohan RaO, "Nonlinear variation of parameters formula for integro-differential equations of Volterra type," J. Math. Anal. Appl., v. 129,1988 , pp. $223-230$.

18. TAKASHI KAMINOGO, "Continuous dependence of solutions for integrodifferential equations with infinite delay," J. Math. Anal. Appl., v. 129, 1988, pp. 307-314.

19. V. Volterra, "Sur la théorie mathématique des phénomènes héréditaires," J. Math. Pures Appl. (9), v. 7, 1928, pp. 249-298 (=Opere Matematiche, v. 5, 1962, pp. 130-169).

20. V. Volterra, Leçons sur la Théorie Mathématique de la Lutte pour la Vie, Gauthier-Villars, Paris, 1931.

21. V. VOLTERRA, "The general equations of biological strife in the case of historical actions," Proc. Edinburgh Math. Soc. (2), v. 6, 1939, pp. 4-10 (=Opere Matematiche, v. 6, 1962, pp. 496-502).

22. G. F. WEbB, Theory of Nonlinear Age-Dependent Population Dynamics, Dekker, New York and Basel, 1985. 